\title{
Tonsil Part
}

National Cancer Institute

\section{Source}

National Cancer Institute. Tonsil Part. NCI Thesaurus. Code C38632.

Any component of the two organs situated in the throat on either side of the narrow passage from the mouth to the pharynx that are composed of lymphoid tissues. 